\title{
Hemosiderin deposition in lymph nodes of patients with plasma cell-type Castleman disease
}

\author{
Yanyan Han, ${ }^{1)}$ Takuro Igawa, ${ }^{1)}$ Kyohei Ogino, ${ }^{2)}$ Asami Nishikori, ${ }^{3)}$ Yuka Gion, \\ Tadashi Yoshino, ${ }^{1)}$ Yasuharu Sato ${ }^{3)}$
}

Plasma cell-type Castleman disease (PCD) is a rare idiopathic atypical lymphoproliferative disorder. It is difficult to differentiate between PCD and IgG4-related disease (IgG4-RD) based on histology alone. As PCD often presents with abundant hemosiderin deposition, lymph node lesions obtained from 22 PCD patients and 12 IgG4-RD patients were analyzed using Prussian blue staining to clarify whether hemosiderin deposition is effective in distinguishing between these two diseases. The analysis disclosed that hemosiderin was more densely deposited in PCD than in IgG4-RD. The median number of Prussian blue-positive cells \pm standard deviation (SD) in PCD and IgG4-RD cases was $13 \pm 36$ cells/3HPFs and $4 \pm 8$ cells $/ 3 \mathrm{HPFs}(\mathrm{P}=0.034)$, respectively. In addition, we analyzed the relationship between hemosiderin deposition and levels of serum interleukin (IL)-6, serum C-reactive protein (CRP), and anemia-related biomarkers. We found that hemosiderin deposition was significantly correlated with the level of serum CRP $(\mathrm{P}=0.045)$; however, no significant correlation was observed between hemosiderin deposition and serum IL-6 levels $(P=0.204)$. A non-significant positive correlation was observed between hemosiderin deposition and serum hemoglobin levels $(\mathrm{P}=0.09)$. Furthermore, no significant correlation was observed between hemosiderin deposition and serum iron levels $(\mathrm{P}=0.799)$. In conclusion, hemosiderin deposition characteristically observed in $\mathrm{PCD}$ may be related to the inflammatory aggressiveness of the disease and could be used for its differential diagnosis.

Keywords: hemosiderin deposition; plasma cell-type Castleman disease; IgG4-related disease; serum IL-6; serum C-reactive protein

\section{INTRODUCTION}

Castleman disease (CD) is a rare idiopathic atypical lymphoproliferative disorder, ${ }^{1}$ with two major histological variants, designated hyaline vascular (HV-CD) and plasma cell (PCD), according to histopathological findings of the affected lesions. ${ }^{2,3}$ The typical characteristics associated with HV-CD include concentric proliferation of mantle zone lymphocytes with hyalinized vascular proliferation in germinal centers and interfollicular areas. ${ }^{3}$ In contrast, PCD is characterized by dense mature plasma cell proliferation in expanded interfollicular areas. ${ }^{3}$ PCD patients often present with systemic manifestations, including fever with abnormal laboratory findings such as anemia, hypoalbuminemia, high C-reactive protein (CRP), and hypergammaglobulinemia. ${ }^{4}$ These characteristics are believed to be caused by dysregulated overproduction of interleukin (IL)-6, which is a pleiotropic cytokine that regulates immune responses.

IgG4-related disease (IgG4-RD) is a recently recognized systemic syndrome characterized by mass-forming lesions and a high serum IgG4 level. ${ }^{6}$ The disease involves fibrosis and severe lymphoplasmacytic infiltration with abundant IgG4-positive cells in various organs, including lymph nodes. ${ }^{7,8}$ IgG4-related lymphadenopathy often lacks fibrosis and phlebitis, in contrast to other lesions affected in IgG4-RD ${ }^{8,9}$ Five histological subtypes have been described: multicentric Castleman-like (type I), reactive follicular hyperplasia-type (type II), interfollicular expansion and immunoblastosis (type III), progressively transformed germinal centers (PTGC)-type (type IV), and inflammatory pseudotumor-like (type $\mathrm{V})$. $^{8,10}$

As both PCD and IgG4-RD are multi-organ disorders with similar histological features of a plasma cell-rich inflammatory infiltrate, it is sometimes difficult to histologically

\footnotetext{
Received: October 9, 2019. Revised: November 17, 2019. Accepted: December 6, 2019. J-STAGE Advance Published: February 8, 2020

DOI: $10.3960 /$ jslrt.19037

${ }^{1}$ Department of Pathology, Okayama University Graduate School of Medicine, Dentistry, and Pharmaceutical Sciences, Okayama, Japan, ${ }^{2}$ Department of Pathology, Fukuyama City Hospital, Fukuyama, Japan, ${ }^{3}$ Division of Pathophysiology, Okayama University Graduate School of Health Sciences, Okayama, Japan

Corresponding author: Dr. Takuro Igawa, Department of Pathology, Okayama University Graduate School of Medicine, Dentistry, and Pharmaceutical Sciences, 2-5-1, Shikata-cho,

Kita-ku, Okayama 700-8558, Japan. E-mail: igawa-t@okayama-u.ac.jp

Copyright (C) 2020 The Japanese Society for Lymphoreticular Tissue Research

(cc) BY-NC-SA This work is licensed under a Creative Commons Attribution-NonCommercial-ShareAlike 4.0 International License.
} 
differentiate PCD from IgG4-RD. ${ }^{11}$ Furthermore, PCD frequently presents with variable lesions infiltrated by large numbers of IgG4-positive plasma cells, accompanied by high serum IgG4 levels. ${ }^{11,12}$

Moreover, hemosiderin deposition has regularly been observed in lymph node lesions of PCD patients. Thus, we assessed the usefulness of hemosiderin deposition in differentiating between PCD and IgG4-RD.

\section{METHODS AND MATERIALS}

\section{Patient selection}

Tissue specimens of lymph nodes were examined from 22 patients with PCD and 12 patients with IgG4-RD. All patients were retrieved from the surgical pathology consultation files of the Department of Pathology, Okayama University, Japan. The study protocol was approved by the Institutional Review Board of Okayama University, Okayama, Japan.

PCD patients with available laboratory data were examined. They were diagnosed based on clinical, laboratory, and pathological findings. The PCD patients consisted of 15 males and seven females aged 35 to 68 years (mean $=52.18$ years). In addition, 14 and seven patients presented with multiple and localized lymph node swelling, respectively. From the 22 PCD patients, nine inguinal, seven cervical, three axillary, one intra-abdominal, one supraclavicular, and one mediastinum lymph node biopsy specimen were examined. Extra nodal lesions were also detected in 14. Laboratory data are summarized in Table 1.

IgG4-RD patients diagnosed according to a previous report consisted of six males and six females aged 36 to 82 years $($ mean $=60.8$ years $) .^{7}$ Of the 12 patients, three presented with systemic lymph node swelling and nine with localized lymph node swelling. Furthermore, six cervical, one axillary, one mediastinum, one inguinal, one submaxillary, one supraclavicular, and one parotid lymph node biopsy specimen were examined. Histological subtypes of these IgG4-related lymphadenopathy samples consisted of five cases of type I, two cases of type II, and five cases of type IV. Extra nodal lesions were also detected in four patients. Laboratory data are summarized in Table 1.

\section{Histological examination}

Surgically biopsied lymph node specimens were fixed in $10 \%$ formaldehyde and embedded in paraffin. Serial 4- $\mu$ m-thick sections were cut from paraffin-embedded tissue blocks, and stained with hematoxylin and eosin (HE).

\section{Prussian blue staining}

Non-stained 4- $\mu$ m-thick sections were deparaffinized with $100 \%$ xylene, followed by replacement of xylene with $100 \%$ alcohol, and finally washed with distilled water. The specimens were treated with a solution containing a mixture of potassium ferrocyanide and hydrochloric acid for $30 \mathrm{~min}-$ utes, and washed with distilled water. They were then treated with Kernechtrot for 5 minutes and washed with distilled water. They were dehydrated with $100 \%$ alcohol, cleared with $100 \%$ xylene, and finally covered with a coverslip and water-soluble mounting medium. The number of Prussian blue-positive cells was counted in areas exhibiting the highest density of Prussian blue-positive cells in three high-power fields (3HPFs).

\section{Statistical analysis}

Data are shown as the median value \pm standard deviation (SD). Comparisons between two groups were performed by a non-parametric version of the Mann-Whitney U test. Spearman rank-based correlation coefficients were used to estimate the correlation between two continuous variables, and corresponding P-values were calculated. All data were

Table 1. Laboratory data

\begin{tabular}{|c|c|c|c|c|}
\hline Variable & $\mathrm{PCD}(\operatorname{median} \pm \mathrm{SD})$ & $\operatorname{IgG} 4-\mathrm{RD}(\operatorname{median} \pm \mathrm{SD})$ & $\mathrm{P}$ & References (range) \\
\hline Number & 22 & 12 & - & - \\
\hline Age & $51.00 \pm 9.73$ & $62.00 \pm 11.99$ & 0.044 & - \\
\hline Sex & $7 \mathrm{~F} / 15 \mathrm{M}$ & $6 \mathrm{~F} / 6 \mathrm{M}$ & - & - \\
\hline $\mathrm{WBC}[\mu \mathrm{L}]$ & $6570.00 \pm 2139.27 \dagger$ & $6445 \pm 1723.69 \$$ & 0.308 & $3900-9800$ \\
\hline $\mathrm{RBC}\left[\times 10^{4} / \mu \mathrm{L}\right]$ & $392 \pm 69.99 \dagger$ & $437.5 \pm 45.01 \ddagger$ & 0.195 & M:420-554/F:384-488 \\
\hline $\mathrm{Hb}[\mathrm{g} / \mathrm{dl}]$ & $11.1 \pm 1.79 \dagger$ & $13.25 \pm 1.50 \ddagger$ & $<0.001$ & M:13.5-17.6/F:11.3-15.2 \\
\hline $\mathrm{Fe}[\mathrm{ug} / \mathrm{dl}]$ & $29 \pm 15.36 \dagger$ & $78 \pm 15 \ddagger$ & 0.041 & $80-140$ \\
\hline Plt $[\times 10000]$ & $38.8 \pm 13.4 \dagger$ & $20.75 \pm 4.82 \ddagger$ & $<0.001$ & $12.7-35.6$ \\
\hline Alb [g/dl] & $2.8 \pm 0.83 \dagger$ & $4.1 \pm 0.93 \ddagger$ & 0.088 & $3.9-4.9$ \\
\hline $\mathrm{CRP}[\mathrm{mg} / \mathrm{dl}]$ & $5.68 \pm 3.12 \dagger$ & $0.08 \pm 0.99 \ddagger$ & $<0.001$ & $<0.30$ \\
\hline $\operatorname{IgG}[\mathrm{mg} / \mathrm{dl}]$ & $4453 \pm 1416.51 \dagger$ & $1577.35 \pm 1973.37 \ddagger$ & 0.003 & $870-1700$ \\
\hline $\operatorname{IgG} 4[\mathrm{mg} / \mathrm{dl}]$ & $563.5 \pm 442.36 \dagger$ & $326 \pm 783.82 \ddagger$ & 0.706 & $4.8-105$ \\
\hline IL-6 $[\mathrm{pg} / \mathrm{mL}]$ & $16.1 \pm 12.09 \dagger$ & $2.3 \$$ & - & $<4.0$ \\
\hline
\end{tabular}

$\mathrm{Hb}$, hemoglobin; Fe, iron; Plt, platelet; Alb, albumin; CRP, C-reactive protein; IL-6, interleukin-6; IgG4-RD, IgG4-related disease; PCD, plasma cell-type Castleman disease; RBC, red blood cell; WBC, white blood cell.

$\dagger$ WBC, RBC, Hb, Fe, Plt, Alb, CRP, IgG, IgG4, and IL-6 were available for 21, 16, 21, 13, 21, 16, 21, 21, 17, and 16 patients, respectively.

WBC, RBC, Hb, Fe, Plt, Alb, CRP, IgG, IgG4, and IL-6 were available for 12, 12, 11, 2, 12, 9, 11, 10, 9, and 1 patient, respectively. 
analyzed using SPSS software v.21.0 (SPSS Inc., Chicago, IL, USA). Results with $\mathrm{P}<0.05$ were regarded as significant.

\section{RESULTS}

\section{Hemosiderin deposition}

Lymph node specimens from both PCD and the five cases of Type I IgG4-related lymphadenopathy showed atrophic to hyperplastic germinal centers and expanded interfollicular areas with sheets of plasma cells. Two cases of Type II IgG4-related lymphadenopathy exhibited hyperplastic germinal centers and mature plasma cell infiltration in interfollicular areas. Five cases of Type IV IgG4-related lymphadenopathy showed follicular hyperplasia with PTGC; abundant plasma cells were found to infiltrate the germinal centers, and many eosinophils were observed in the interfollicular zone. Macrophages were also observed in the interfollicular areas of PCD and IgG4-RD. Following HE staining, hemosiderin was detected in macrophages as a granular yellow-brown pigment in PCD, whereas little hemosiderin was detected in IgG4-RD (Fig. 1). Prussian blue staining clearly revealed hemosiderin deposition. Prussian blue-positive cells were detected in interfollicular areas of PCD specimens, whereas
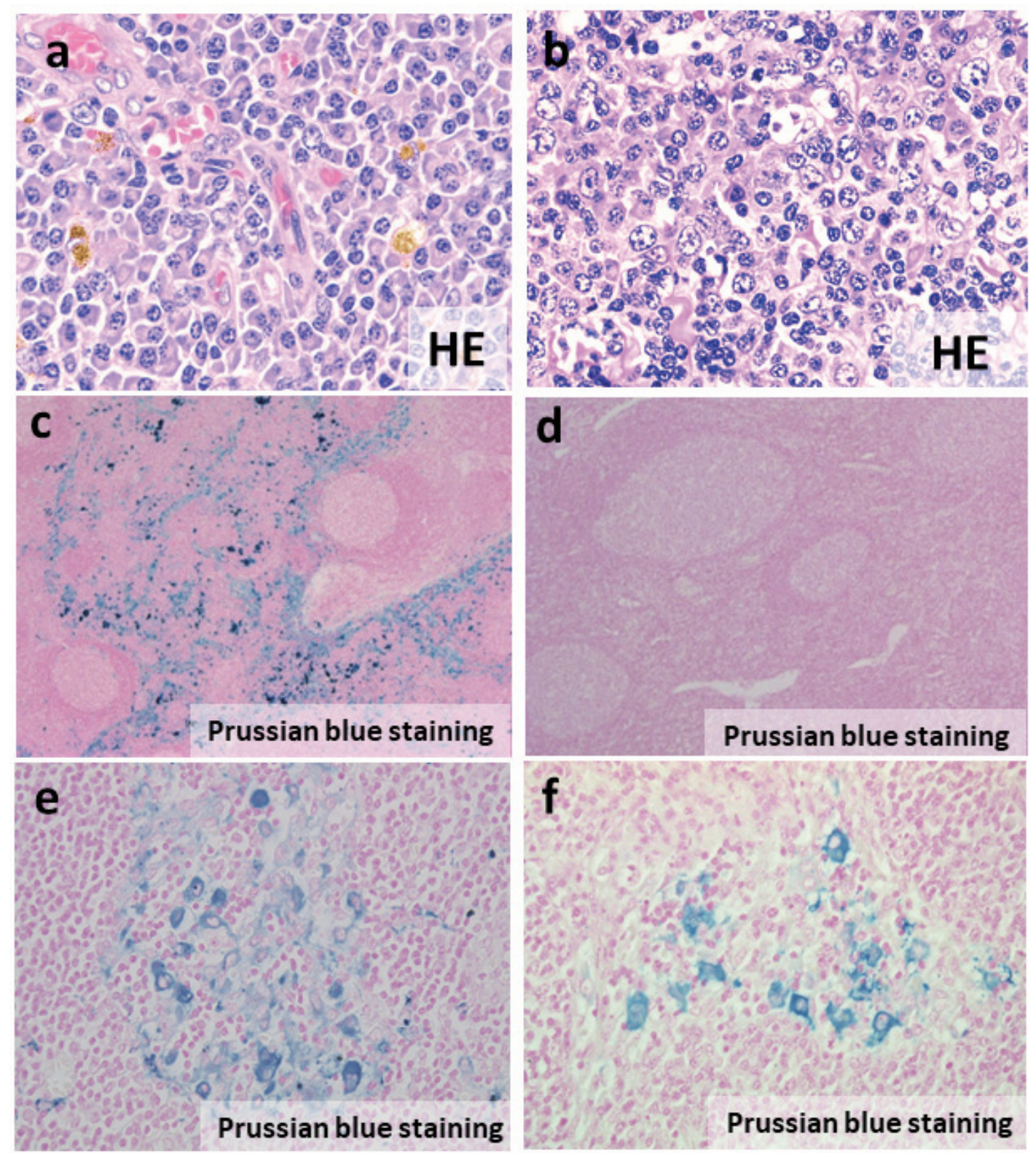

Fig. 1. Histological examination and Prussian blue staining

$(\boldsymbol{a}, \boldsymbol{c}, \boldsymbol{e})$ Plasma cell-type Castleman disease (PCD) $(\boldsymbol{b}, \boldsymbol{d}, \boldsymbol{f})$ IgG4-related disease (IgG4-RD) $(\boldsymbol{a}, \boldsymbol{b})$ HE staining $(\boldsymbol{c}, \boldsymbol{d}, \boldsymbol{e}, \boldsymbol{f})$ Prussian blue staining. $(\boldsymbol{a}, \boldsymbol{b})$ Hemosiderin deposition was observed in PCD, but not in IgG4-RD. (c) Prussian blue staining revealed hemosiderin deposition in the interfollicular area of PCD specimens. $(\boldsymbol{d})$ Hemosiderin deposition was not detected or was rarely observed in typical cases of IgG4-RD. (e, $\boldsymbol{f})$ Hemosiderin deposition was observed in macrophages. ( $f$ ) Prussian blue-positive cells in IgG4-RD were not morphologically different from those in PCD. 
no or very few Prussian blue-positive cells were seen in IgG4-RD cases (Fig. 1). The median number of Prussian blue-positive cells in PCD and IgG4-RD cases was $13 \pm 36$ cells/3HPFs (range: $0-113$ cells/3HPFs) and $4 \pm 8$ cells/3HPFs (range: $1-27$ cells $/ 3$ HPFs) (Fig. 2$)(\mathrm{P}=0.034)$. The median number of Prussian blue-positive cells in Type I IgG4-RD cases [12 \pm 10 cells/3HPFs (range: $1-27$ cells/3HPFs)] was higher than that in Type II $[5 \pm 2$ cells/3HPFs (range: $3-6$ cells/3HPFs) $(\mathrm{P}=0.439)]$ and Type IV [ $3 \pm 5$ cells $/ 3$ HPFs (range: $2-15$ cells $/ 3$ HPFs $)(P=0.341)]$.

\section{Association between hemosiderin deposition and anemia- related markers}

An exploratory correlation analysis was performed to examine the relationship between hemosiderin deposition and anemia-related markers such as serum iron levels and serum hemoglobin values. Serum iron levels were examined in 13 patients with PCD (range: 16-69 $\mu \mathrm{g} / \mathrm{dl}$ ). No correlation was observed between hemosiderin deposition and serum iron levels ( $\mathrm{r}=-0.078, \mathrm{P}=0.799)$ (Fig. 3). The median serum hemoglobin value in PCD was $11.10 \pm 1.79(\mathrm{~g} / \mathrm{dl})$ (range: $7.1-13.2 \mathrm{~g} / \mathrm{dl}$ ). A non-significant positive correlation was observed between serum hemoglobin levels and number of Prussian blue-positive cells in 3HPFs $(r=0.3793, \mathrm{P}=0.09)$ (Fig. 3).

\section{Relationship between hemosiderin deposition and serum IL-6/CRP}

Serum IL-6 levels were high in all available PCD cases $(\mathrm{n}=16)$. The median serum IL-6 level in PCD was $16.1 \pm$ $12.09(\mathrm{pg} / \mathrm{mL})$. A correlation was observed between serum IL-6 levels and the number of Prussian blue-positive cells in 3HPFs; however, it was not significant $(\mathrm{r}=0.3352, \mathrm{P}=$ 0.204) (Fig. 3).

The correlation between serum CRP levels and hemosiderin deposition in PCD was assessed in PCD cases $(n=21)$.

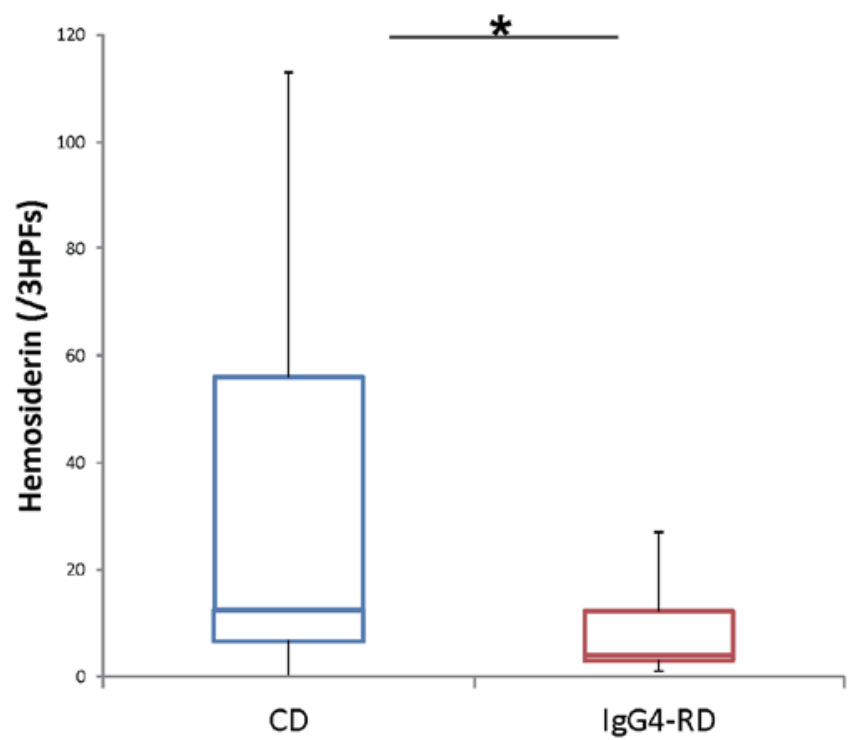

Fig. 2. Hemosiderin deposition counts in PCD and IgG4-RD Prussian blue-positive cells in PCD and IgG4-RD are shown in boxplots with whiskers, representing minimum to maximum values. Differences between the two groups were evaluated by the MannWhitney $\mathrm{U}$ test $(* \mathrm{P}<0.05)$. More Prussian blue-positive cells were observed in PCD than in IgG4-RD. a

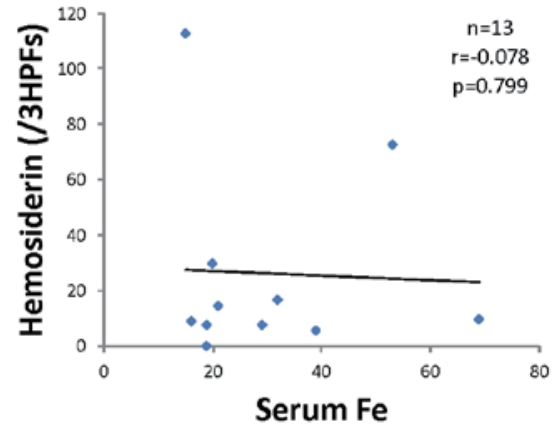

c

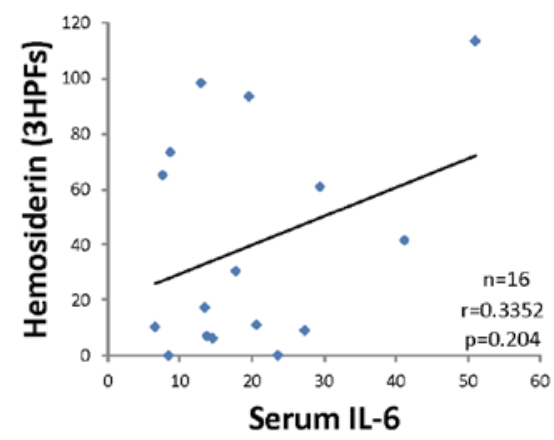

b

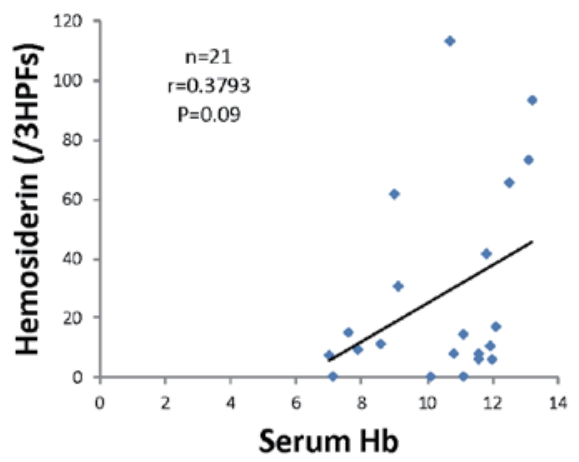

d

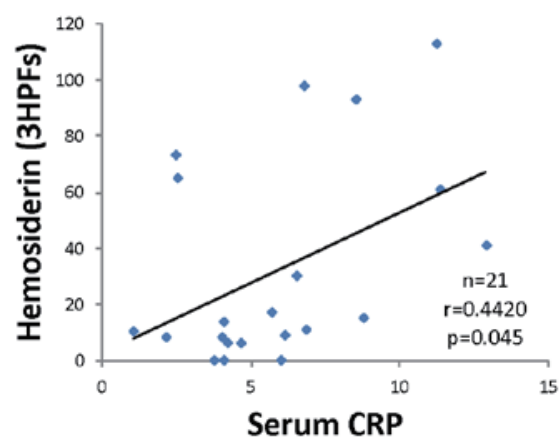

Fig. 3. Relationship between hemosiderin deposition and serum Fe/Hb/IL-6/ CRP in PCD Correlation between number of Prussian blue-positive cells in three high-power fields (3HPFs) and serum $(\boldsymbol{a})$ iron, $(\boldsymbol{b})$ hemoglobin, $(\boldsymbol{c})$ IL-6, and $(\boldsymbol{d})$ CRP. The positive correlation between CRP levels and hemosiderin in 3HPFs was significant $(\mathrm{P}<0.05)$. 
In all the cases, serum CRP levels exhibited levels above the reference range. The positive correlation between CRP levels and hemosiderin deposition in 3HPFs was significant $(\mathrm{r}=$ 0.4420, $\mathrm{P}=0.045$ ) (Fig. 3).

\section{DISCUSSION}

In this study, hemosiderin deposition was densely observed in PCD patient samples, whereas sparse or no deposition was seen in IgG4-RD. It is sometimes difficult to distinguish PCD from IgG4-RD, as they have similar histological features including plasma cell infiltration in the interfollicular areas; additionally, PCD patient lymph nodes can also exhibit abundant infiltration of IgG4-positive cells. ${ }^{11,12}$ Specific characteristics have been reported as useful in differentiating PCD from IgG4-RD, including the presence of IgA-positive cells in PCD, and eosinophilic infiltration observed in IgG4-RD. ${ }^{11,13}$ Furthermore, hemosiderin deposition appears to be useful in differentiating between the two diseases. A useful approach to distinguish PCD from IgG4-RD may, therefore, be to evaluate a combination of factors including hemosiderin deposition, presence of IgApositive cells, and eosinophil infiltration. Currently, however, the differential diagnosis between the two diseases requires comprehensive diagnostic procedures based on not only histopathological findings, but also laboratory data and clinical findings. ${ }^{3}$

Hemosiderin is an iron-storage complex observed as a granular yellow-brown degradation product on HE staining. The deposit is tiny and often inconspicuous, unless using Prussian blue staining. Hemosiderin originates from the breakdown of hemoglobin or from an abnormal metabolic pathway of ferritin. ${ }^{14}$ When damaged or destroyed erythrocytes leave a ruptured blood vessel, they may release hemoglobin into the extracellular space. The damaged red blood cells or the released hemoglobin can then be phagocytosed by macrophages. ${ }^{14}$ As hepcidin is known to not only inhibit intestinal iron absorption, but also block iron release from macrophages, ${ }^{15}$ hemosiderin deposition can be increased when hepcidin is upregulated. Moreover, as IL-6 is known to be a key inducer of hepatic hepcidin synthesis ${ }^{16}$ overexpression of hepcidin is likely to be associated with hemosiderin deposition in the tissues of patients with PCD.

Overproduction of IL-6 has been suggested to drive PCD development. ${ }^{5}$ Although the positive relationship between hemosiderin deposition and serum IL-6 levels in our study was not significant, a positive correlation between serum CRP levels and hemosiderin deposition was observed. As CRP expression is induced by IL-6 during inflammation, CRP has been considered as a relevant biomarker for indirect measurement of IL-6 bioactivity. ${ }^{17,18}$ Thus, we propose hemosiderin deposition to be associated with PCD disease activity.

It is well documented that PCD causes microcytic anemia, mimicking iron-deficiency anemia. ${ }^{1}$ The PCD patients in our study also presented with anemia and low serum iron levels. Although PCD patients with severe anemia were speculated to have high disease activity and show abundant hemosiderin deposition, our data showed no significant relationship between hemosiderin deposition and serum iron or hemoglobin levels. This might be because anemia counteracts the effects of IL-6 on hepcidin production. Plasma erythropoietin (EPO) has been reported to mediate hepcidin suppression in response to altered hematopoietic activity. ${ }^{19}$ As anemia deteriorates, EPO concentrations increase, leading to the downregulation of IL-6-induced hepcidin production. ${ }^{20}$

\section{CONCLUSION}

Hemosiderin was clearly deposited in lymph node specimens from patients with PCD. This finding may be useful in differentiating between PCD and IgG4-RD. Hepcidin may be closely related to hemosiderin deposition in PCD. Our study suggested that PCD disease activity is positively correlated with hemosiderin deposition.

\section{CONFLICT OF INTEREST}

The authors have no competing interests to declare.

\section{REFERENCES}

1 Fajgenbaum DC. Novel insights and therapeutic approaches in idiopathic multicentric Castleman disease. Blood. 2018; 132 : 2323-2330.

2 Keller AR, Hochholzer L, Castleman B. Hyaline-vascular and plasma-cell types of giant lymph node hyperplasia of the mediastinum and other locations. Cancer. 1972; $29: 670-683$.

3 Fajgenbaum DC, Uldrick TS, Bagg A, et al. International, evidence-based consensus diagnostic criteria for HHV-8-negative/ idiopathic multicentric Castleman disease. Blood. 2017; 129 : 1646-1657.

4 Frizzera G, Peterson BA, Bayrd ED, Goldman A. A systemic lymphoproliferative disorder with morphologic features of Castleman's disease: clinical findings and clinicopathologic correlations in 15 patients. J Clin Oncol. 1985; 3 : 1202-1216.

5 Yoshizaki K, Murayama S, Ito H, Koga T. The role of interleukin-6 in Castleman Disease. Hematol Oncol Clin North Am. 2018; $32: 23-36$

6 Stone JH, Zen Y, Deshpande V. IgG4-Related Disease. N Engl J Med. 2012; 366 : 539-551.

7 Deshpande V, Zen Y, Chan JKC, et al. Consensus statement on the pathology of IgG4-related disease. Mod Pathol. 2012; 25 : 1181-1192.

8 Sato Y, Yoshino T. IgG4-Related Lymphadenopathy. Int J Rheumatol. 2012; 2012 : 572539.

9 Cheuk W, Yuen H, Chu S, et al. Lymphadenopathy of IgG4related sclerosing disease. Am J Surg Pathol. 2008; 32 : 671-681.

10 Sato Y, Notohara K, Kojima M, et al. IgG4-related disease: historical overview and pathology of hematological disorders. Pathol Int. 2010; 60 : 247-258.

11 Manabe A, Igawa T, Takeuchi M, et al. Immunohistochemical analysis of IgA expression differentiates IgG4-related disease 
from plasma cell-type Castleman disease. Med Mol Morphol. 2017; $50: 34-41$.

12 Zoshima T, Yamada K, Hara S, et al. Multicentric Castleman disease with tubulointerstitial nephritis mimicking IgG4-related disease: two case reports. Am J Surg Pathol. 2016; 40 : 495-501.

13 Sun C, Xu G, Lin J. Comparison of IgG4-related lymphadenopathy and multicentric Castleman's disease: a retrospective study. Clin Lab. 2018; 64 : 1671-1678.

14 Wang Y, LV J, Ma X, et al. Specific hemosiderin deposition in spleen induced by a low dose of cisplatin: altered iron metabolism and its implication as an acute hemosiderin formation model. Curr Drug Metab. 2010; 11 : 507-515.

15 Sonkar SK, Singh NK, Sonkar GK, et al. Association of hepcidin and anemia in early chronic kidney disease. Saudi J Kidney Dis Transpl. 2019; $30: 315-324$.

16 Wrighting DM, Andrews NC. Interleukin-6 induces hepcidin expression through STAT3. Blood. 2006; 108 : 3204-3209.
17 Kurzrock R, Voorhees PM, Casper C, et al. A phase I, openlabel study of siltuximab, an anti-IL-6 monoclonal antibody, in patients with B-cell non-Hodgkin lymphoma, multiple myeloma, or Castleman disease. Clin Cancer Res. 2013; 19 : 3659-3670.

18 Puchalski T, Prabhakar U, Jiao Q, Berns B, Davis HM. Pharmacokinetic and pharmacodynamic modeling of an antiinterleukin-6 chimeric monoclonal antibody (siltuximab) in patients with metastatic renal cell carcinoma. Clin Cancer Res. $2010 ; 16: 1652-1661$.

19 Song SNJ, Tomosugi N, Kawabata H, et al. Down-regulation of hepcidin resulting from long-term treatment with an anti-IL-6 receptor antibody (tocilizumab) improves anemia of inflammation in multicentric Castleman disease. Blood. 2010; 116 : 3627-3634.

20 Ganz T. Erythropoietic regulators of iron metabolism. Free Radic Biol Med. 2019; 133 : 69-74. 